\title{
Endogenous Endophthalmitis Associated with Facial Cellulitis after a Tongue Bite
}

\author{
Min Ho Kang ${ }^{1,2}$, Mincheol Seong ${ }^{1,2}$, Ju Hak Lee ${ }^{3}$, Hee Yoon Cho ${ }^{1,2^{*}}$ \\ ${ }^{1}$ Department of Ophthalmology, Hanyang University College of Medicine, Seoul, Korea; ${ }^{2}$ Department of Ophthalmology, Hanyang \\ University Guri Hospital, Guri, Korea; ${ }^{3}$ Department of Nephrology, Hanyang University College of Medicine, Seoul, Korea. \\ Email: *hycho@hanyang.ac.kr
}

Received March 20 ${ }^{\text {th }}$, 2012; revised April 25 ${ }^{\text {th }}, 2012$; accepted May $18^{\text {th }}, 2012$

\begin{abstract}
Endogenous endophthalmitis is an intraocular infection that results from hematogenous spread of organisms from a distant source of infection. Facial cellulitis is rarely reported as a focus of infection. We report a case of 51-year-old unconscious woman presenting with fever, facial swelling, and decreased visual acuity secondary to facial cellulitis, endogenous endophthalmitis and end-stage renal disease (ESRD). Generally systemic antibiotics in endophthalmitis have low efficacy because of the blood-ocular barrier. The management of endophthalmitis begins with intravitreal antibiotic injections and if the response is not favorable to do a vitrectomy. Twelve hours after the intravitreal antibiotic injection, vitrectomy was considered because of worsening of the vitreal cloudiness. However, the patient's general medical condition precluded vitrectomy. We experienced successful treatment with intravitreal antibiotic injection and continuous intravenous antibiotic administration because of the breakdown of the blood-ocular barrier due to ocular inflammation, especially in the setting of systemic vascular and fluid homeostatic changes.
\end{abstract}

Keywords: Endogenous Endophthalmitis; Facial Cellulitis; End-Stage Renal Disease; Ocular Blood Barrier

\section{Introduction}

Endogenous endophthalmitis is an intraocular infection that results from hematogenous spread of organisms from a distant source of infection, such as a liver abscess or endocarditis $[1,2]$. According to a recent review, approximately $2 \% \sim 6 \%$ of endophthalmitis cases are caused by endogenous infection [3]. Endogenous endophthalmitis is associated with underlying medical conditions, such as diabetes, cardiac disease, and malignancy, in up to $90 \%$ of patients. The common foci of infection are urinary tract infection, septic arthritis, pneumonia, and endocarditis [4]. Orbital and periorbital cellulitis were reported as causes of endogenous endophthalmitis [5,6], but facial cellulitis is rarely reported as a focus of infection leading to endogenous endophthalmitis [7]. We observed a case of endogenous endophthalmitis with facial cellulitis in a patient with end-stage renal disease (ESRD) and achieved improvement with bilateral intravitreal (vancomycin, ceftazidime) and intravenous antibiotics (vancomycin, meropenem).

\section{Case Report}

A 51-year-old woman was admitted to the emergency

${ }^{*}$ Corresponding author. department (ED) with fever, chills, and facial swelling. (Figure 1). On physical examination, vital signs were blood pressure 210/110 $\mathrm{mmHg}$ in the supine position, pulse rate $94 / \mathrm{min}$, respiratory rate $28 / \mathrm{min}$, and temperature $36.6^{\circ} \mathrm{C}$. The patient weighed $68 \mathrm{~kg}$ and was drowsy and delirious but able to be awakened. She had peripheral edema and jugular venous distension. Initial laboratory data in the ED were white blood cell (WBC) count 17300/ $\mathrm{mm}^{3}$ (segmentation ratio: 96\%), hemoglobin $5.8 \mathrm{~g} / \mathrm{dL}$,

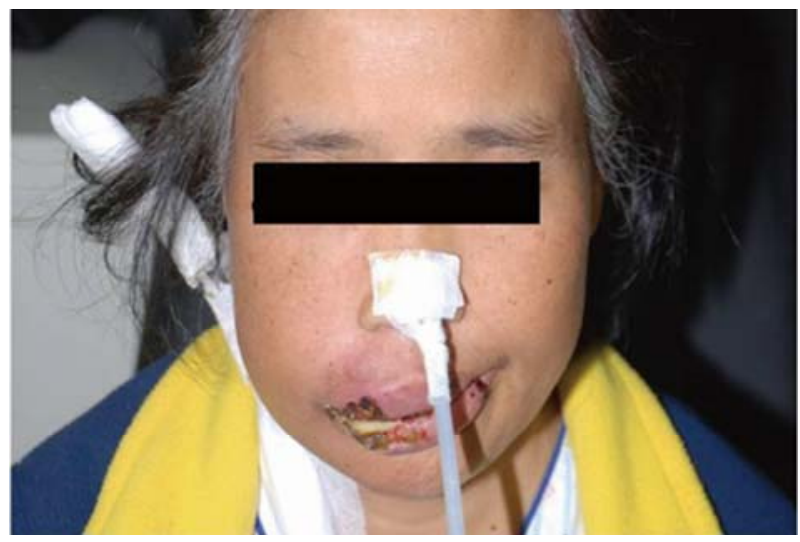

Figure 1. Right facial cellulitis after a tongue bite. A double lumen catheter was inserted in the right jugular vein for hemodialysis. 
and hematocrit $17.1 \%$. Serum sodium was $130 \mathrm{mEq} / \mathrm{L}$, potassium $5.6 \mathrm{mEq} / \mathrm{L}$, chloride $90 \mathrm{mEq} / \mathrm{L}$, total carbon dioxide $6 \mathrm{mEq} / \mathrm{L}$, blood urea nitrogen $227 \mathrm{mg} / \mathrm{dL}$, and creatinine $21.4 \mathrm{mg} / \mathrm{dL}$. Abdominal ultrasonography revealed shrunken kidneys with bilateral thinning of the renal cortex. These findings led to a presumptive diagnosis of chronic renal failure rather than acute kidney injury. She had no known underlying disease; however, four days before visiting the ED, she had a seizure that lasted 10 minutes during which she bit her tongue. The seizure was thought to be a manifestation of uremia. The day after the seizure, she had a fever and lost consciousness. During hospital admission, Staphylococcus aureus were found in the blood culture. Given the diagnosis of sepsis and acute renal failure, intravenous vancomycin (Vancocin, Parmalink, Korea) and carbapenem (Meropenem, Sumitomo, Japan) were administered, and emergent hemodialysis was performed daily. Facial CT demonstrated facial cellulitis sparing the orbit. Three days later, the patient regained consciousness and complained of decreased visual acuity. There was no history of ocular trauma, infection, or surgery. Ophthalmic examination of both eyes showed a best corrected visual acuity of 20/200 and normal intraocular pressure. Slit lamp examination revealed a moderate anterior chamber reaction with keratic precipitates in both eyes. The fundus was obscured by vitreous cloudiness with multiple discrete white retinal lesions and hard exudates (Figure 2). Intraocular cultures (anterior chamber tapping, vitreal aspiration) were obtained, and intravitreal vancomycin $(1.0 \mathrm{mg} / 0.1$ $\mathrm{ml}$, Vancocin, Parmalink, Korea) and ceftazidime (2.25 $\mathrm{mg} / 0.1 \mathrm{ml}$, Tazime, Hanmi, Korea) injections were performed due to the diagnosis of endogenous endophthalmitis. Additionally, intravenous vancomycin, meropenem and hemodialysis were continued for 14 days to treat the patient's sepsis and end-stage renal failure. Culture and gram smear of vitreous revealed Gram-positive cocci, Staphylococcus aureus compatible with the causative organism isolated from blood culture.

Twelve hours after the intravitreal antibiotic injection, vitrectomy was considered because of worsening of the vitreal cloudiness. However, the patient's general medical condition precluded vitrectomy. Therefore, we planned to observe and continue the intravenous antibiotics. One day after the intravitreal injection, visual acuity improved, and the fundus findings became clearer. Visual acuity was 20/25 in both eyes 8 days after the injection. Fundoscopy revealed diminished white retina lesions and improved clarity of the vitreous (Figure 3). At the final follow-up, 3 years after the injection, there was no relapse, or recurrence. She has received cataract surgery additionally due to cataract secondary to intraocular inflammation in both eyes. Her final visual acuity was 20/20 in both eyes.

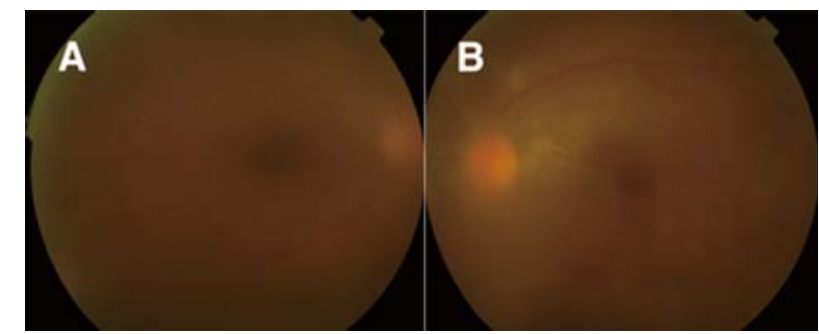

Figure 2. Fundus photographs at first examination. (A) Photographs of the right eye revealed vitreous cloudiness; (B) In the left eye, vitreous cloudiness was less severe; however, hard exudates and multiple discrete lesions were observed.

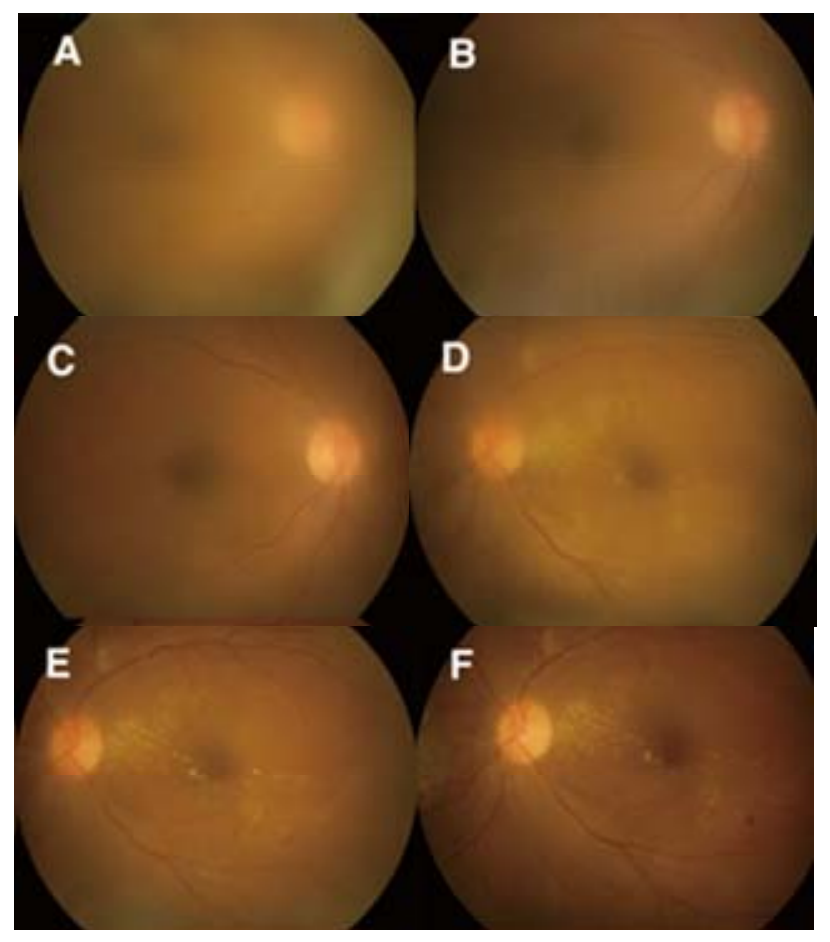

Figure 3. Serial fundus photographs after intravitreal antibiotics injection. Upper pictures are of the right eye on days 2, 5, and 8 after intravitreal antibiotic injection (A, B, C). Lower pictures are of the left eye on days 2, 5, and 8 after the injection (D, E, F). Fundus images demonstrated gradual vitreous clearing.

\section{Discussion}

According to prior reports, most endogenous endophthalmitis develops from pneumonia, hepatobiliary disease, myocarditis, or meningitis [3]. However, facial cellulitis as a focus of endogenous endophthalmitis is rare. Facial cellulitis usually appears more rapidly than other deep infections, so treatment is performed earlier. For this reason, endogenous endophthalmitis associated with facial cellulitis is relatively rare. However, facial cellulitis can be a direct or indirect causative infection. The indirect pathway involves distant spread through the blood stream 
via the internal jugular vein. Microorganisms are then able to spread through the heart to the internal carotid artery and ophthalmic artery. Furthermore, they can follow a retrograde pathway toward the cavernous sinus of the skull, establishing thrombophlebitis in the facial vessels [4]. These anatomical characteristics explain how facial cellulitis can be a primary infection site of endogenous endophthalmitis.

We also considered that a severe uremic condition due to ESRD could contribute to the development of endogenous endophthalmitis because severe uremia generates an immunosuppressive state. Although hemodialysis (HD) can provide a direct infection route for normal skin flora. [8]. HD may have had a positive effect on the treatment of endogenous endophthalmitis in this case because the severe uremia that contributed to an immunosuppressive state was well treated by HD. Many ophthalmologists think that systemic antibiotics in endophthalmitis have low efficacy because of the blood-ocular barrier [9]. A major difference between this case and previous reports was successful treatment with intravitreal antibiotic injection and continuous intravenous antibiotic administration. The patient's ESRD could have potentially contributed to improvement of her disease. In ESRD, low serum protein reduces colloid osmotic pressure, and renal-induced hypertension increases hydrostatic pressure [8]. Therefore, a higher pressure gradient may be induced between the blood to the retina. Although we did not evaluate her previous fundoscopy, we expect that the patient had hypertensive retinopathy due to uncontrolled hypertension and a very low estimated GFR, $2 \mathrm{~mL} / \mathrm{min} / 1.73$ $\mathrm{m}^{2}$. According to a recent report, a low estimated GFR is associated with a much higher incidence of fundus pathology, similar to that in hypertensive retinopathy [10]. In addition to hypertensive retinopathy, inflammation of the retina and retinal vasculature in endophthalmitis disturbs the inner and outer blood-retinal barrier [9]. These changes may increase retinal vascular permeability, which may aid systemic antibiotics in more effectively reaching the retina. In endogenous endophthalmitis, Greenwald et al. proposed that the breakdown of the blood-ocular barrier by ocular infection allows adequate penetration of systemic antibiotics into the vitreous cavity $[9,11]$. Intravenous imipenem also achieved excellent penetration of the vitreous in a prior report [12]. It is possible that all of these factors might produce a synergistic effect.

However, as shown in many studies, intravenous antibiotics cannot be the main treatment of endogenous endophthalmitis. In this exceptional case, we were able to treat endogenous endophthalmitis with intravenous and intravitreal antibiotics due to the patient's special condition, which included ESRD. Intravitreal injection of antibiotics remains important because the intravitreal route provides immediate and high drug concentrations [12].

\section{REFERENCES}

[1] S. R. Smith, A. J. Kroll, P. L. Lou and E. A. Ryan, "Endogenous Bacterial and Fungal Endophthalmitis,” International Ophthalmology Clinics, Vol. 47, No. 2, 2007, pp. 173-183. doi:10.1097/IIO.0b013e31803778f7

[2] R. Argelich, N. Ibáñez-Flores, J. Bardavio, A. BurésJelstrup, G. García-Segarra, R. Coll-Colell, V. Cuadrado and F. Fernández-Monrás, "Orbital Cellulitis and Endogenous Endophthalmitis Secondary to Proteus Mirabilis Cholecystitis," Diagnostic Microbiology and Infectious Disease, Vol. 64, No. 4, 2009, pp. 442-444. doi:10.1016/j.diagmicrobio.2009.04.014

[3] T. L. Jackson, S. J. Eykyn, E. M. Graham and M. R. Stanford, "Endogenous Bacterial Endophthalmitis: A 17-Year Prospective Series and Review of 267 Reported Cases," Survey of Ophthalmology, Vol. 48, No. 4, 2003, pp. 403423. doi:10.1016/S0039-6257(03)00054-7

[4] T. Ness, K. Pelz and L. L. Hansen, "Endogenous Endophthalmitis: Microorganisms, Disposition and Prognosis," Acta Ophthalmologica Scandinavica, Vol. 85, No. 8, 2007, pp. 852-856. doi:10.1111/j.1600-0420.2007.00982.x

[5] A. Ebneter, L. Goold and J. S. Gilhotra, “A Rare Case of Endogenous Streptococcus Group C Endophthalmitis Associated with Cellulitis,” Eye, Vol. 25, No. 9, 2011, pp. 1239-1240. doi:10.1038/eye.2011.124

[6] E. Peker, E. Cagan, M. Dogan, A. Kilic, H. Caksen and O. Yesilmen, "Periorbital Cellulitis Caused by Bacillus Thuringiensis,” European Journal of Ophthalmology, Vol. 20, No. 1, 2010, pp. 243-245.

[7] J. C. de Vincnete-Rodrigez, "Maxillofacial Cellulites," Medicina Oral, Patologia Oral y Cirugia Bucal, Vol. 9 2004, pp. 133-138.

[8] K. G. Smith, B. U. Ihle and W. J. Heriot, "Metastatic Endophthalmitis in Dialysis Patients," American Journal of Nephrology, Vol. 15, No. 1, 1995, pp. 78-81. doi:10.1159/000168805

[9] J. Cunha-Vaz, “The Blood-Ocular Barriers,” Survey of Ophthalmology, Vol. 23, No. 5, 1979, pp. 279-296. doi:10.1016/0039-6257(79)90158-9

[10] J. E. Grunwald, J. Alexander, M. Maguire, R. Whittock, C. Parker, K. McWilliams, J. C. Lo, R. Townsend, C. A. Gadegbeku, J. P. Lash, J. C. Fink, M. Rahman, H. Feldman, J. Kusek and A. Ojo, “CRIC Study Group, Prevalence of Ocular Fundus Pathology in Patients with Chronic Kidney Disease," Clinical Journal of American Society Nephrology, Vol. 5, No. 5, 2010, pp. 867-873. doi:10.2215/CJN.08271109

[11] M. J. Greenwald, L. G. Wohl and C. H. Sell, "Metastatic Bacterial Endophthalmitis: A Contemporary Reappraisal," Survey of Ophthalmology, Vol. 31, No. 2, 1986, pp. 81-101. doi:10.1016/0039-6257(86)90076-7

[12] M. Engelbert, L. G. Miohl and C. H. Sell, ”Intravitreal Vancomycin and Amikacin versus Intravenous Imipenem in the Treatment of Experimental Staphylococcus Aureus 
Endophthalmitis," Graefes Arch Clinical Experiment

Ophthalmology, Vol. 242, No. 4, 2004, pp. 313-320.

doi:10.1007/s00417-003-0767-9 